\title{
EIF2AK2-related Neurodevelopmental Disorder With Leukoencephalopathy, Developmental Delay, and Episodic Neurologic Regression Mimics Pelizaeus-Merzbacher Disease
}

Daniel G. Calame, MD, PhD, Meagan Hainlen, MD, Danielle Takacs, MD, Leah Ferrante, MD, Kayla Pence, MD, Lisa T. Emrick, MD, and Hsiao-Tuan Chao, MD, PhD

Neurol Genet 2021;7:e539. doi:10.1212/NXG.0000000000000539

\section{Abstract}

\section{Objective}

To demonstrate that de novo missense single nucleotide variants (SNVs) in EIF2AK2 cause a neurodevelopmental disorder with leukoencephalopathy resembling Pelizaeus-Merzbacher disease (PMD).

\section{Methods}

A retrospective chart review was performed of 2 unrelated males evaluated at a single institution with de novo EIF2AK2 SNVs identified by clinical exome sequencing (ES). Clinical and radiographic data were reviewed and summarized.

\section{Results}

Both individuals presented in the first year of life with concern for seizures and developmental delay. Common clinical findings included horizontal and/or pendular nystagmus during infancy, axial hypotonia, appendicular hypertonia, spasticity, and episodic neurologic regression with febrile viral illnesses. MRI of the brain demonstrated severely delayed myelination in infancy. A hypomyelinating pattern was confirmed on serial imaging at age 4 years for proband 1. In proband 2, repeat imaging at age 13 months confirmed persistent delayed myelination. These clinical and radiographic features led to a strong suspicion of PMD. However, neither PLP1 copy number variants nor pathogenic SNVs were detected by chromosomal microarray and trio ES, respectively. Reanalysis of trio ES identified heterozygous de novo EIF2AK2 missense variant c.290C $>\mathrm{T}$ (p.Ser97Phe) in proband 1 and c.326C $>\mathrm{T}$ (p.Ala109Val) in proband 2 .

\section{Conclusions}

The autosomal dominant EIF2AK2-related leukoencephalopathy, developmental delay, and episodic neurologic regression syndrome should be considered in the differential diagnosis for PMD and other hypomyelinating leukodystrophies (HLDs). A characteristic history of developmental regression with febrile illnesses may help distinguish it from other HLDs.
Correspondence

Dr. Calame

daniel.calame@bcm.edu; Dr. Chao hc140077@bcm.edu

From the Division of Neurology and Developmental Neuroscience (D.G.C., D.T., L.F., K.P., L.T.E., H.-T.C.), Department of Pediatrics, BCM, Houston, TX; Texas Children's Hospital (D.G.C., D.T., L.F., K.P., L.T.E., H.-T.C.), Houston, TX; Department of Neurology and Neurotherapeutics (M.H.), UTSW, Dallas, TX; Department of Molecular and Human Genetics (L.T.E., H.T.C.), BCM, Houston, TX; Department of Neuroscience (H.-T.C.), BCM, Houston, TX; Program in Development (H.-T.C.), Disease Models, and Therapeutics, BCM, Houston, TX; MCNair Medical Institute (H.-T.C.), The Robert and Janice McNair Foundation, Houston, TX; and Jan and Dan Duncan Neurological Research Institute (H.-T.C.), Texas Children's Hospital, Houston, TX. 


\section{Glossary}

ACMG = American College of Medical Genetics; EEG = electroencephalogram; HLD = hypomyelinating leukodystrophy; ISR = integrated stress response; LEUDEN = leukoencephalopathy, developmental delay, and episodic neurologic regression; OFC = occipital-frontal head circumference; $\mathbf{O M I M}=$ Online Mendelian Inheritance in Man; PMD = Pelizaeus-Merzbacher disease; $\mathbf{V M W}=$ vanishing white matter.

Pelizaeus-Merzbacher disease (PMD) (\#312080) is an X-linked recessive hypomyelinating leukodystrophy $(\mathrm{HLD})^{1-4}$ caused by copy number or single nucleotide variants in Proteolipid Protein 1 (PLP1) located on Xq22.2. PLP1 encodes a major myelin membrane protein in the CNS and functions in the development and maintenance of myelin sheaths, as well as oligodendrocyte development and axonal survival. ${ }^{4}$ Classic PMD is characterized by infantile-onset pendular nystagmus that may have horizontal and rotatory components, head bobbing, axial hypotonia, and developmental delay. The nystagmus typically disappears as the individual matures, but appendicular hypertonia, ataxia, spasticity, epilepsy, and involuntary movements develop as the disease progresses. Most individuals with PMD acquire speech, and some acquire independent ambulation, but progressive dysarthria and spastic paraparesis can lead to loss of these developmental milestones. A key finding in PMD is a pattern consistent with CNS hypomyelination on MRI. ${ }^{5}$

Although PMD accounts for the majority of HLD cases, a significant proportion (20\%-40\%) of clinically diagnosed PMD lack identifiable $P L P 1$ variants. ${ }^{6-8}$ As of May 2020, 19 genes are indexed in the Online Mendelian Inheritance in Man (OMIM) in association with HLD, several of which share overlapping features with PMD. Therefore, the recognition of disorders that mimic PMD features is key for identifying cardinal clinical or radiographic features to guide diagnostic testing and prognostication. Early recognition may facilitate access to clinical trials and targeted therapeutic interventions such as neural stem cell transplantation. ${ }^{9}$ In 2020, heterozygous missense variants in Eukaryotic Initiation Factor 2 Alpha Kinase 2 (EIF2AK2), a gene located on chromosome 2q22.2 and encoding a protein serine/threonine kinase, were shown to cause the newly recognized leukoencephalopathy, developmental delay, and episodic neurologic regression syndrome (LEUDEN, OMIM \#618877). ${ }^{10}$ The autosomal dominant EIF2AK2-related LEUDEN syndrome is characterized by delayed development, delayed myelination, hypomyelination, ataxia, hypotonia, hypertonia, neurologic regression, and comorbidities including epilepsy, autistic features, and movement disorders. Here, we provide a comprehensive expanded clinical and radiographic description of 2 unrelated individuals with de novo EIF2AK2 missense variants, which establish EIF2AK2-related neurodevelopmental and leukoencephalopathy disorder as a differential diagnosis for PMD.

\section{Methods}

Clinical data were obtained after written informed consent was obtained in accordance with the ethical standards of the institutional review board on human research.

\section{Data Availability}

Deidentified data regarding molecular and clinical findings are available on request.

\section{Proband 1}

A male of European descent presented at age 9 months for neurologic evaluation of spells concerning for seizures. $\mathrm{He}$ had recurrent unprovoked episodes consisting of bilateral arm and leg extension and grunting lasting 30 seconds or less followed by irritability and fatigue. Physical examination was significant for axial hypotonia and persistent horizontal nystagmus. Occipital-frontal head circumference (OFC), height, and weight were $44.5 \mathrm{~cm}(12 \%, \mathrm{Z}=-1.17), 70 \mathrm{~cm}(12.75 \%, \mathrm{Z}$ $=-1.14)$, and $7.6 \mathrm{~kg}(5.98 \%, Z=-1.56)$, respectively. He was born at term following an uncomplicated pregnancy and delivery. He exhibited mild early developmental delay with ability to coo, but no babbling, and able to sit with anterior propping but was not crawling or pulling to stand by age 12 months. Horizontal bilateral nystagmus and head bobbing were noted at age 2 months. He had a healthy brother. Family history was noncontributory and without consanguinity. Electroencephalogram (EEG) demonstrated rare spike and wave activity in the midline and right frontocentral region during sleep (figure 1A). He was diagnosed with focal-onset tonic motor seizures and was started on levetiracetam with good response. Dilated fundoscopic examination showed normal-appearing retina and optic disc.

He developed progressive appendicular hypertonia requiring baclofen and botulinum toxin injections by age 4 years. Motor development was significant for crawling at age 14 months and pulling to stand at age 3 years. He acquired a few hand signs but as of 5 years of age had not acquired spoken language and was unable to follow simple commands. His nystagmus gradually decreased in frequency and was rarely seen by age 3 years. He had failure to thrive, and a G-tube was placed at age 3 years to provide supplemental nutrition overnight. There was no evidence of dysphagia on swallow study at age 3 years. At age 4 years, he developed jaw tightness, further limiting his oral intake, and was treated with an uptitration of baclofen.

At age 5 years, he was hospitalized due to hypovolemic vs septic shock and acute respiratory failure related to human metapneumovirus pneumonia. Before hospitalization, he was able to crawl, pull to stand, drink from a cup independently, and ate food by mouth. Afterward, he was unable to crawl, could no longer hold a cup on his own, and struggled with oral intake. Retrospectively, he had similar but less profound 
Figure 1 Abnormal Electroencephalography of EIF2AK2-related Leukoencephalopathy, Developmental Delay, and Episodic Neurologic Regression Syndrome

A. Proband 1, rare midline and right frontocentral spike and wave activity

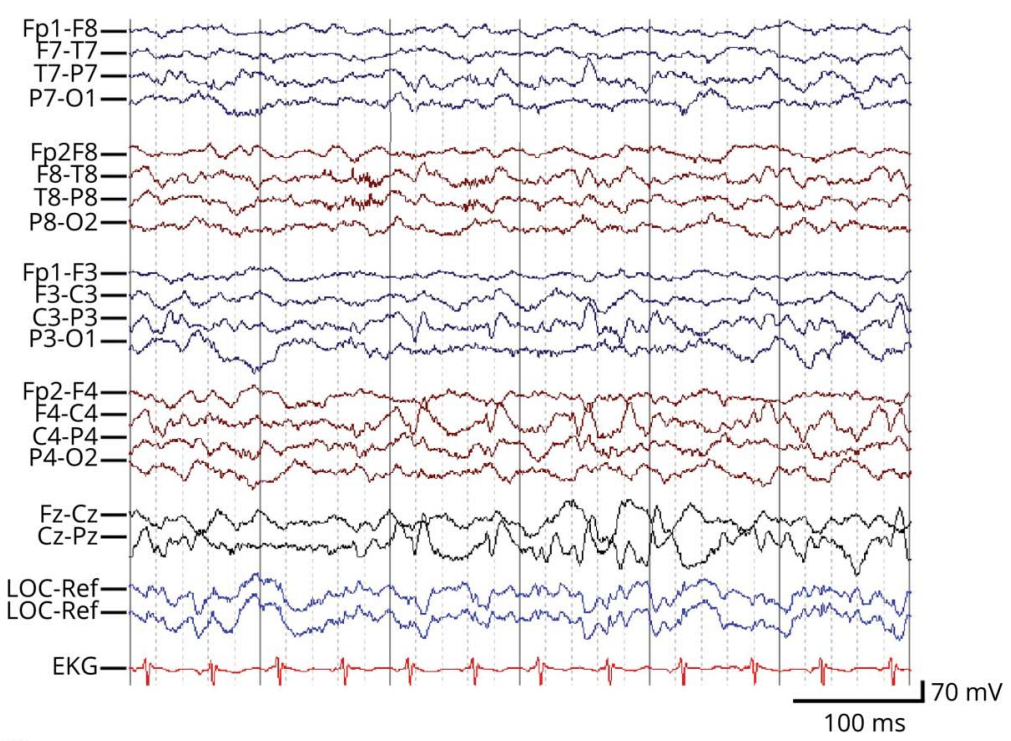

B. Proband 2, diffuse slowing

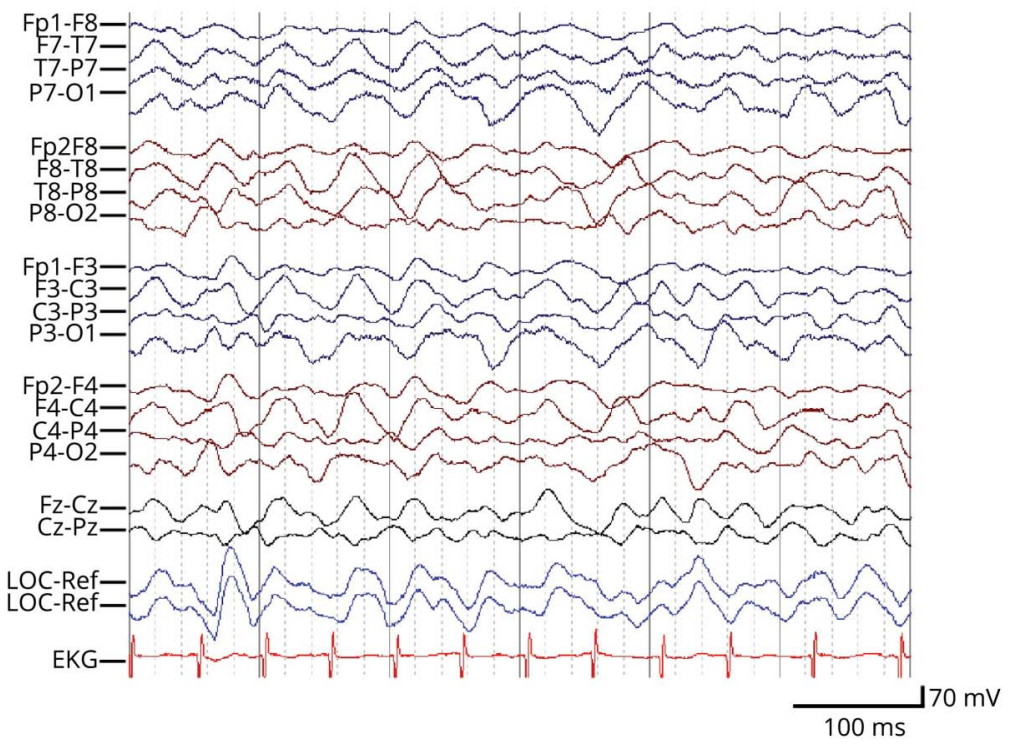

Representative EEG tracings for probands 1 and 2. (A) Proband 1 exhibited rare midline/right frontocentral spike and wave in sleep. (B) Proband 2 exhibited diffuse slowing while awake during an episode of developmental regression at age 13 months. Multiple staring spells were captured during the video EEG without electrographic correlate (not shown). EEG electrodes: prefrontal (Fp), frontal (F), temporal (T), parietal $(P)$, occipital $(O)$, central $(C)$, electrode placed on midline sagittal plane (FpZ, Fz, Cz, and Oz), electrooculogram electrodes (LOC-Ref), and ECG (EKG). episodic regressions with prior febrile illnesses per parental reports. He also exhibited hypoglycemia during several presumed viral gastrointestinal illnesses and was diagnosed with idiopathic ketotic hypoglycemia. At last examination at age 5 years, he exhibited axial hypotonia and appendicular hypertonia, hyperreflexia, bradykinesia, and dysmetria. His head circumference at age 4 years and 8 months was $49 \mathrm{~cm}(5.34 \%$, $\mathrm{Z}=-1.61)$.

Diagnostic evaluation included normal metabolic studies (lactate, ammonia, acylcarnitine profile, plasma amino acids, urine organic acids, and very-long-chain fatty acids) and chromosomal microarray. Targeted PLP1 duplication/deletion analysis was unrevealing. Pathogenic PLP1 variants were not identified on trio ES. A single maternally inherited variant of unknown significance was identified in vacuolar protein sorting 11 (VPS11), c.1982C > T (p.Thr661Met). VPS11 causes autosomal recessive HLD12 (OMIM: \#616683), and therefore, a single rare variant would unlikely be responsible for his condition. No other variants in genes associated with HLD were identified. Reanalysis of the trio ES identified a de novo missense variant in EIF2AK2, c.290C $>\mathrm{T}$ (p.Ser97Phe) that is not found in gnomAD. ${ }^{10-12} \mathrm{The}$ variant is poorly conserved with variable in silico predictions. According to the American College of Medical Genetics (ACMG) guidelines EIF2AK2, c.290C $>\mathrm{T}$ (p.Ser97Phe) is classified as likely pathogenic (PS2, PM2, and BP4). ${ }^{13}$ 
Figure 2 Delayed Myelination or Hypomyelinating Pattern in EIF2AK2-related Leukoencephalopathy, Developmental Delay, and Episodic Neurologic Regression Syndrome

Proband 1, EIF2AK2 p.Ser97Phe, 9-months-old
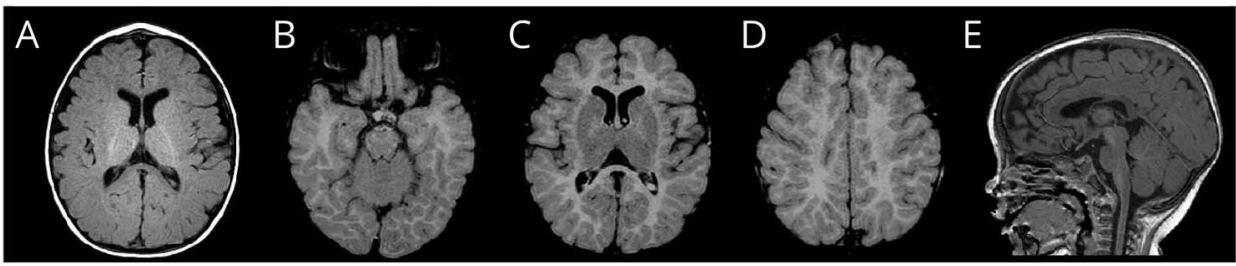

Proband 1, EIF2AK2 p.Ser97Phe, 4-years-old

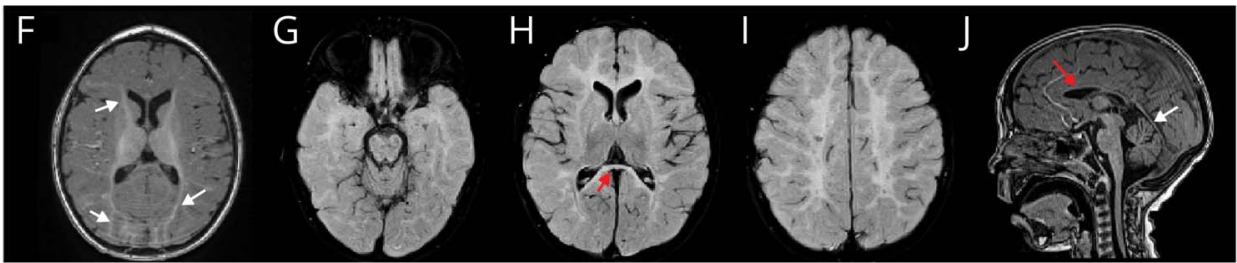

Proband 2, EIF2AK2 p.Ala109Val, 7-months-old

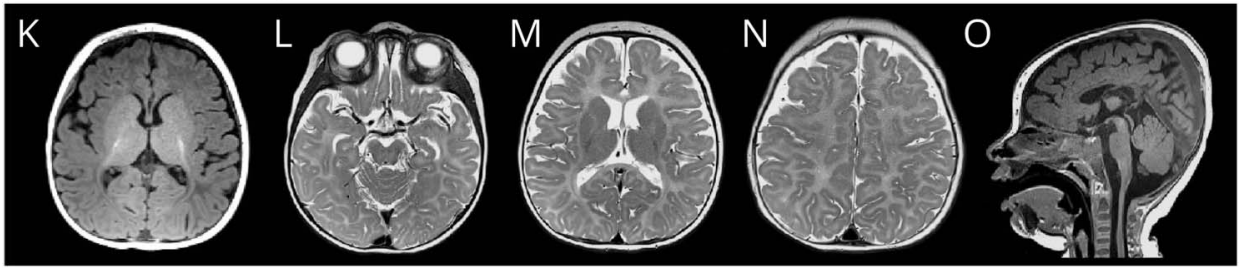

Proband 2, EIF2AK2 p.Ala109Val, 13-months-old

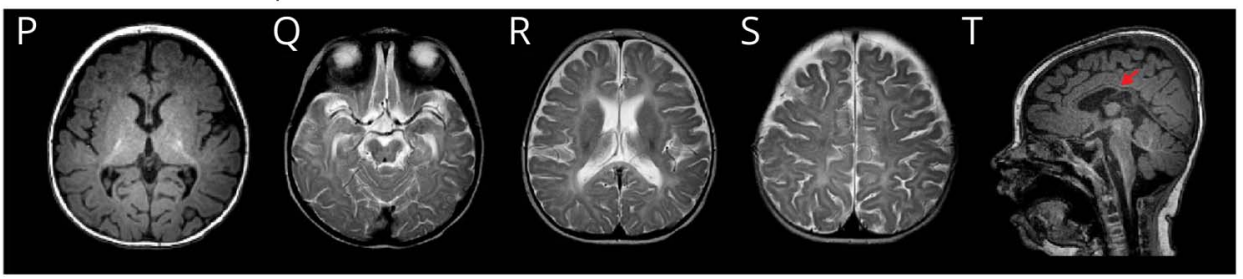

Representative serial brain MRIs for proband 1 at age 9 months (A-E) and 4 years (F-J) and for proband 2 at age 7 months (K-O) and 13 months (P-T). Representative axial T1 (A, F, K, and P), sagittal $T 1(E, J, O, T)$ weighted, axial fluid-attenuated inversion recovery (FLAIR) (B, C, D, G, H, and I), and axial T2-weighted (L, $M, N, Q, R$, and S) images are shown. Myelination in proband 1 at age 9 months (A-E) was limited to the posterior limb of the internal capsule on T1-weighted imaging, with little normal myelination on T2-weighted imaging. By age 4 years (F-J), myelination progressed on T1. weighted imaging to include the anterior limb of the internal capsule and deep frontal and occipital white matter (white arrows), but white matter remained diffuse and symmetrically FLAIR hyperintense. Corpus callosum atrophy and cerebellar atrophy are indicated by red and white arrows, respectively. Myelination in proband 2 at age 7 months $(\mathrm{K}-\mathrm{O})$ was similarly limited to the posterior limb of the internal capsule on T1-weighted imaging, with little normal myelination on T2-weighted imaging. Repeat imaging at age 13 months (P-T) showed minimal progression of myelination and corpus callosum thinning (red arrow).
Because of the neurologic regression following febrile illnesses, proband 1 underwent clinical immunology evaluation. He was found to have mild to moderate $\mathrm{T}$-cell lymphopenia $\left(546 \times 10^{3} / \mu \mathrm{L} \mathrm{CD}^{+} \mathrm{CD}^{+} \mathrm{T}\right.$ cells [normal 700-2,200] and $377 \times 10^{3} / \mu \mathrm{L} \mathrm{CD}^{+} \mathrm{CD}^{+} \mathrm{T}$ cells [normal 490-1,300]), normal $\mathrm{B}$ and NK cell populations, and borderline low Streptococcus pneumoniae antibody titers.

\section{Proband 2}

A male of Moroccan descent presented at age 7 months for neurologic evaluation of spells concerning for seizure activity. The unprovoked spells consisted of arm flexion and leg extension with apnea. There were no clear behavioral changes or fatigue following these spells. Physical examination was significant for head bobbing and axial hypotonia with horizontal and pendular nystagmus. His OFC, height, and weight were $41 \mathrm{~cm}$ $(<1 \%, Z=-2.67), 73 \mathrm{~cm}(91 \%, Z=1.36)$, and $7.825 \mathrm{~kg}(23 \%$, $\mathrm{Z}=-0.76)$, respectively. He had motor delays and at age 7 months was not rolling over or sitting. His birth history was significant for intrauterine growth restriction, oligohydramnios, and preterm birth at 36-week gestation secondary to maternal bleeding. He was admitted to the neonatal intensive care unit due to hypoglycemia and apneas and discharged home at age 2 weeks. Family history was significant for a healthy older brother and consanguinity. His parents were first cousins once removed. Routine EEG showed normal background and no epileptiform activity. Dilated fundoscopic examination showed normal-appearing retina and optic disc.

At age 13 months, he experienced severe developmental regression with a febrile illness due to influenza A. Before the illness, he was sitting independently, crawling, standing, and taking steps with support, had head control, and was babbling. During the illness, he developed frequent staring spells, ataxia, and progressive lethargy. Hospital examination revealed loss of head control, no reaction to visual confrontation, axial hypotonia, and appendicular hypertonia more prominent in the lower extremities. Prolonged video EEG showed diffuse background slowing and captured multiple staring spells without electrographic correlate (figure 1C). He developed dysphagia, and a swallow study showed aspiration with thin liquids. Two weeks later, at hospital discharge, he could sit with support but continued to have limited response to verbal or physical stimuli. A month following discharge, at age 14 
Pelizaeus-Merzbacher-like disease, 2-years-old

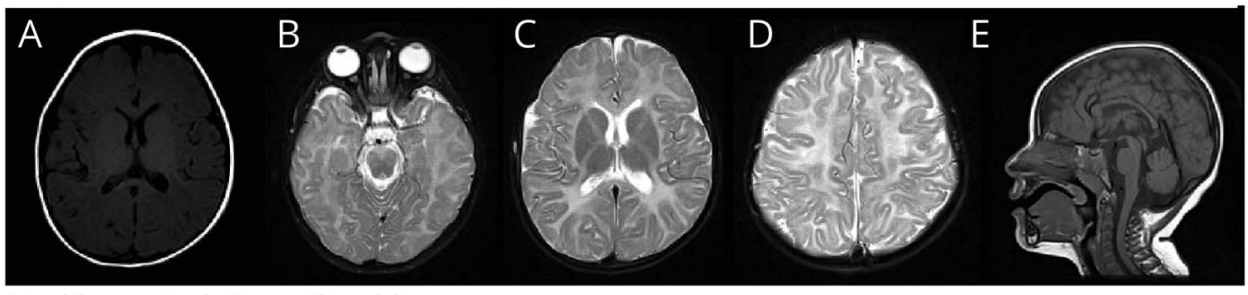

Healthy control, 8-months-old

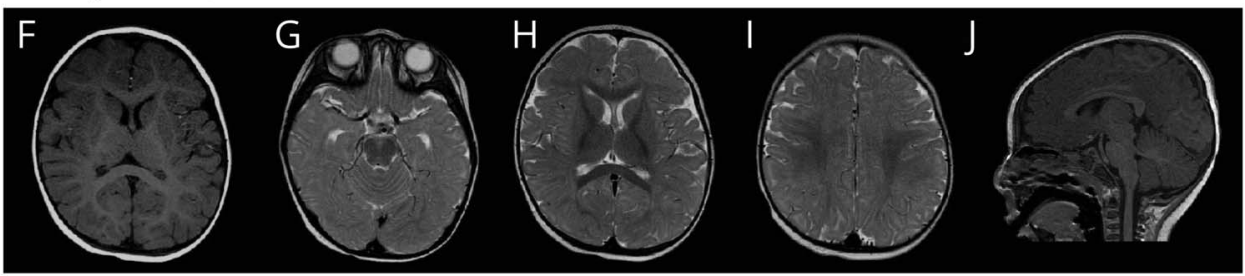

Healthy control, 7-years-old

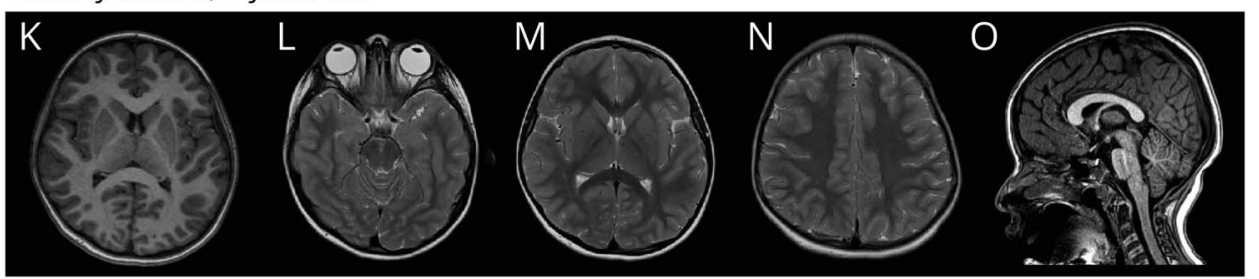

Representative brain MRI images of a patient with Pelizaeus-Merzbacherlike disease (PMLD) at age 2 years $(A-E)$, a healthy control at age 8 months (F-J), and a healthy control at age 7 years $(\mathrm{K}-\mathrm{O})$. Representative axial $\mathrm{T} 1(\mathrm{~A}, \mathrm{~F}$, and $\mathrm{K})$, sagittal $\mathrm{T} 1(\mathrm{E}, \mathrm{J}$, and $\mathrm{O})$ weighted, and axial T2-weighted (B-D, G-I, and L-N) images are shown. A complete lack of myelination is evident in the patient with PMLD, with the white matter uniformly T1 hypointense or isointense and $\mathrm{T} 2$ hyperintense relative to the cortex (A-E). In an 8-month-old healthy control, the white matter appears largely T1 hyperintense (F). The genu of the corpus callosum, anterior and posterior limbs of the internal capsule, perirolandic gyri, and some of the deep occipital white matter are $\mathrm{T} 2$ hypointense, indicating completed myelination (G-I). Imaging in a 7-year-old healthy control (K-O) demonstrates completed myelination ( $\mathrm{T} 1$ hyperintense and $\mathrm{T} 2$ hypointense) that occurs around age 24 months.

months, he had improved head control and started to sit independently and crawl. His nystagmus resolved by age 16 months. By age 22 months, he could stand with assistance but exhibited truncal ataxia. He had failure to thrive due to persistent dysphagia.

Diagnostic evaluation included normal metabolic studies (lactate, ammonia, acylcarnitine profile, plasma amino acids, and urine organic acids). Chromosomal microarray array did not identify any known pathogenic copy number variations. However, a $47-\mathrm{Mb}$ region was identified with absence of heterozygosity on chromosome $17(17 \mathrm{p} 11.2 \mathrm{q} 24.1)$ in a region not associated with known genetic disorders. Trio ES failed to identify variants in PLP1 or other genes associated with HLD. Reanalysis of the trio ES revealed a de novo EIF2AK2 missense variant, c.326C $>\mathrm{T}$ (p.Ala109Val) that is not present in gnomAD and is poorly conserved with variable in silico predictions. ${ }^{10-12}$ It is classified as likely pathogenic (PS2, PM2, and BP4) by ACMG guidelines. ${ }^{13}$

\section{Radiologic Features}

Proband 1 was imaged at age 9 months (figure 2, A-E) and 4 years (figure 2, F-J). MRI finding was significant for global delayed myelination at age 9 months. By age 4 years, MRI findings revealed that myelination had progressed on T1weighted imaging (figure $2 \mathrm{~F}$ ) but remained diffusely hypomyelinated with symmetrically hyperintense signal on T2-FLAIR (figure 2, G-I). Additional findings include cerebellar and cortical atrophy and corpus callosum thinning (figure $2 \mathrm{H}$ and $\mathrm{J}$ ). Representative images of a 2-year-old patient with Pelizaeus-Merzbacher-like disease (figure 3, A-E), a healthy 8-month-old (figure 3, F-J), and a healthy 7-yearold (figure $3, \mathrm{~K}-\mathrm{O}$ ) are provided for comparison.

Proband 2 was imaged at age 7 months (figure 2, K-O) and 13 months (figure 2, P-T). MRI finding was significant for global delayed myelination delay at age 7 months. By age 13 months, MRI findings revealed minimal progression in myelination on $\mathrm{T} 1$ or $\mathrm{T} 2$ sequences. Additional findings include cortical atrophy and corpus callosum thinning (figure $2 \mathrm{~T}$ ).

\section{Discussion}

De novo EIF2AK2 missense variants were recently identified as causing a neurodevelopmental disorder characterized by developmental delay, variable leukoencephalopathy, and neurologic decompensation. ${ }^{10}$ EIF2AK2, a member of the eukaryotic translation initiation factor 2-alpha kinase family, regulates the cytoprotective integrated stress response (ISR). ${ }^{14}$ The ISR is activated by numerous stressors including heme deprivation, endoplasmic reticulum stress, oxidative stress, cytokines, and double-stranded RNA. ${ }^{15-18}$ On activation, EIF2AK2 phosphorylates EIF2S1, which in turn acts as a competitive inhibitor of EIF2B and suppresses protein translation. ${ }^{14}$ Variants in genes encoding all 5 EIF2B subunits (EIF2B1, EIF2B2, EIF2B3, EIF2B4, and EIF2B5) are well recognized as the cause of leukoencephalopathy with 
Table A Phenotypic Comparison of EIF2AK2 Probands With Leukoencephalopathy, Developmental Delay, and Episodic Neurologic Regression Syndrome and Classic PMD

\begin{tabular}{|c|c|c|c|c|}
\hline Feature & EIF2AK2 p.Ser97Phe & EIF2AK2 p.Ala109Val & Other reported EIF2AK2 probands ${ }^{10}$ & Classic PMD \\
\hline Nystagmus & + & + & 1 of $6(17 \%)$ & + \\
\hline Hypotonia & + & + & 5 of $6(83 \%)$ & + \\
\hline Head bobbing & + & + & 1 of $6(17 \%)$ & + \\
\hline Spasticity & + & + & 5 of $6(83 \%)$ & + \\
\hline Ataxia & + & + & 5 of $6(83 \%$ & + \\
\hline Developmental delay & + & + & 6 of $6(100 \%)$ & + \\
\hline Delayed myelination & + & + & 6 of $6(100 \%)$ & + \\
\hline Hypomyelinating pattern & + & $?$ & 1 of $6(17 \%)$ & + \\
\hline Dystonia & - & - & 3 of $6(50 \%)$ & $+/-$ \\
\hline Chorea & - & - & 1 of $6(17 \%)$ & $+/-$ \\
\hline Epilepsy & + & - & 2 of $6(33 \%)$ & $+/-$ \\
\hline Microcephaly & - & + & 1 of $6(17 \%)$ & $+/-$ \\
\hline Developmental regression & + & + & 6 of $6(100 \%)$ & - \\
\hline
\end{tabular}

Abbreviation: EIF2AK2 = eukaryotic initiation factor 2-alpha kinase 2.

EIF2AK p.Ser97Phe (proband 1), EIF2AK2 p.Ala109Val (proband 2), PMD = Pelizaeus-Merzbacher disease. + /- indicates a feature that occurs in some but not all individuals with the condition. Hypomyelinating pattern indicates diffuse, confluent white matter T2 hyperintensity and T1 isointensity or hypointensity as seen in PMD.

vanishing white matter (VWM). ${ }^{19}$ Given the shared pathway and tendency toward neurologic regression, it is likely that the 2 disorders (EIF2AK2-related neurodevelopmental disorder and VWM) have a common pathogenic mechanism. ${ }^{10}$ However, from a clinical perspective, the 2 disorders can be distinguished radiographically.

In both presymptomatic and symptomatic individuals with VWM, characteristic findings include diffuse and symmetric white matter $\mathrm{T} 1$ hypointensity and $\mathrm{T} 2$ hyperintensity sparing only the U fibers, outer corpus callosum, internal capsule, and anterior commissure. ${ }^{19}$ Progressive rarefaction and cystic white matter degeneration are observed. ${ }^{19}$ In contrast, imaging findings for the EIF2AK2-related neurodevelopmental disorder range from delayed myelination or a hypomyelinating pattern as described here to more focal leukoencephalopathy with $\mathrm{T} 1$ hyperintensity/isointensity and T2 hyperintensity variably involving the periventricular white matter, inferior cerebellar peduncles, and the dorsal upper cervical cord, medulla, and pons. ${ }^{10}$

Here, we provide expanded phenotypic evidence that recently identified pathogenic EIF2AK2 variants are associated with a clinical presentation mimicking PMD. Typical features of PMD include male sex, infantile onset nystagmus, hypotonia, head bobbing, spastic quadriparesis, ataxia, cognitive impairment, delayed development, and hypomyelination on brain MRI. ${ }^{1-4}$ In its classic form, PMD begins in the first few months of life with nystagmus and hypotonia. Nystagmus is typically pendular but may also have horizontal and/or rotatory components. The nystagmus often diminishes with age as seen in the 2 probands here. Similarly, with age, the initial hypotonia is replaced by hypertonia and spasticity. Movement disorders (such as dystonia and chorea) and epilepsy can also occur. As spasticity progresses, ambulation and other motor functions are gradually lost, but acute regression in the setting of a defined febrile illness is uncommon. The 2 probands described here share many of the cardinal PMD features (table). A hypomyelinating pattern can be established in proband 1 based on his repeat imaging at age 4 years (figure 1 , F-J). As proband 2 was only imaged once after age 12 months, a hypomyelinating pattern cannot be diagnosed until repeat brain MRI is conducted. ${ }^{5}$ However, the unchanged pattern of myelination between age 7 and 13 months is suggestive of a hypomyelinating pattern (figure $1, \mathrm{~K}-\mathrm{T}$ ).

Although developmental delay, hypotonia, spasticity, and ataxia were seen in most individuals with the EIF2AK2-related neurodevelopmental disorder, nystagmus and head bobbing were only seen in 1 of the other 6 probands reported in the initial LEUDEN syndrome cohort (table). ${ }^{10}$ In addition, the degree of leukoencephalopathy was variable, ranging from diffuse white matter abnormalities to more localized areas (e.g., T2 hyperintensity involving the upper cervical cord, dorsal medulla, dorsal pons, and periaqueductal gray matter). ${ }^{19}$ Thus, a larger sample size is required to determine the prevalence of PMD-like presentation in individuals with EIF2AK2-related neurodevelopmental disorder. 


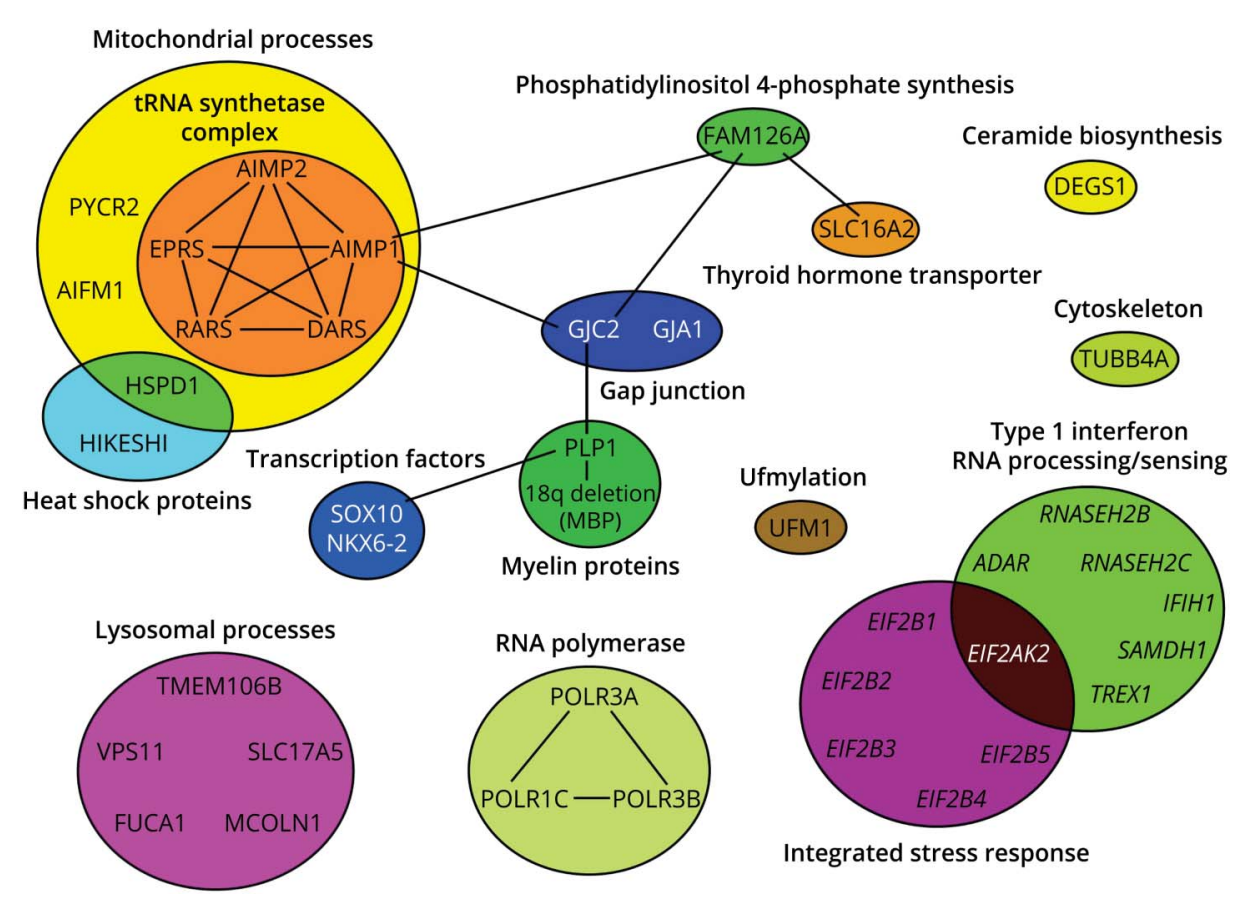

A list of genes responsible for HLD and related disorders was curated from Online Mendelian Inheritance in Man (OMIM), Vanderver et al., ${ }^{20}$ or GeneReviews (ncbi.nlm.nih.gov/books/NBK1116/). Gene functions were identified via OMIM or PubMed, and protein-protein interactions were identified via STRING (string-db.org). Protein-protein interactions are indicated by lines. Genes are clustered in colored circles based biological function with each color representing a functional class (e.g., components of the mitochondrial tRNA synthetase complex, i.e., AIMP1, AIMP2, EPRS, RARS, and DARS, are indicated by orange). The genes responsible for vanishing white matter (in purple: EIF2B1, EIF2B2, EIF2B3, EIF2B4, and EIF2B5) and Aicardi-Gouiterres syndrome (in green: ADAR, RNASEH2B, RNASEH2C, SAMDH1, TREX1, and IFIH1) are italicized to indicate that although hypomyelination is observed in these disorders, they are generally considered distinct from HLDs. The hypomyelinating genes and their respective disease-associated phenotypes with OMIM \#s are PYCR2 (HLD10, \#616420); AIFM1 (spondyloepimetaphyseal dysplasia, X-linked, with hypomyelinating leukodystrophy, \#300232); HSPD1 (HLD4, \#612233); HIKESHI (HLD13, \#616881); AIMP2 (HLD17, \#618006); EPRS (HLD15, \#617951); RARS (HLD9, \#616140); AIMP1 (HLD3, \#260600); DARS (hypomyelination with brainstem and spinal cord involvement and leg spasticity, \#615281); FAM126A (HLD5, \#610532); SLC16A2 (AllanHerndon-Dudley syndrome, \#300523); GJC2 (HLD2, \#608804); GJA1 (oculodentodigital dysplasia, \#164299 and 257850); PLP1 (Pelizaeus-Merzbacher disease, \#312080); MBP (chromosome 18q deletion syndrome, \#601808); SOX10 (peripheral demyelinating neuropathy, central dysmyelination, Waardenburg syndrome, and Hirschsprung disease, \#609136); NKX6-2 (spastic ataxia 8, autosomal recessive, with hypomyelinating leukodystrophy, \#605955); TMEM106B (HLD16, \#617964); VPS11 (HLD12, \#616683); FUCA1 (fucosidosis, \#230000); SLC17A5 (Salla disease, \#604369, and infantile sialic acid storage disease, \#269920); MCOLN1 (mucolipidosis IV, \#252650): POLR3A (HLD7, \#607694); POLR1C (HLD11, \#616494); POLR3B (HLD8, \#614381); UFM1 (HLD14, \#617899); DEGS1 (HLD18, \#618404); and TUBB4A (HLD6, \#612438).

The molecular mechanisms underlying the pathogenesis of HLDs and related disorders remain poorly understood. To explore the relationship between EIF2AK2 and the genes responsible for these conditions, we performed a function and interactome-based analysis of genes implicated in HLDs and related disorders (figure 4). ${ }^{20}$ Although several HLDs involve genes responsible for myelin proteins (PLP1 and MBP in 18q deletion syndrome) or transcription factors regulating oligodendrocyte development (SOX10 and NKX6-2), a wide range of other biological processes are involved, such as RNA polymerase I and III (POLR1C, POLR3A, and POLR3B), lysosomal trafficking and metabolism (VPS11, TMEM106B, SLC17A5, MCOLN1, and FUCA1), and mitochondrial processes
(EPRS, RARS, DARS, AIMP1, AIMP2, PYCR2, AIFM1, and HSPD1). Although EIF2AK2 does not directly overlap on a functional or interactome level with other HLD genes, the same can be said of both DEGS1 (HLD18, OMIM \#618404, ceramide biosynthesis) and TUBB4A (HLD5, OMIM \#612438, cytoskeleton). As mentioned previously, there is a clear overlap between EIF2AK2 and the genes responsible for VWM (EIF2B1, EIF2B2, EIF2B3, EIF2B4, and EIF2B5). In addition, as a double-stranded RNA sensor and component of the type 1 interferon response pathway, a parallel can be drawn between EIF2AK2 and the genes responsible for Aicardi-Goutières syndrome ( $A D A R$, RNASEH2B, RNASEH2C, SAMDH1, TREX1, and IFIH1). Although these 2 conditions are not classified as HLDs, 
hypomyelination can occur in both conditions. Therefore, function and interactome-based models may be useful when evaluating candidate HLD genes in the future.

Distinct clinical and radiographic features aid in distinguishing the different HLDs. For example, the presence of comorbid skeletal dysplasia, congenital cataracts, and the combination of hypodontia and hypogonadotropic hypogonadism distinguishes hypomyelination with spondyloepiphyseal dysplasia (AIFM1), hypomyelination and congenital cataract (FAM126A), and 4H leukodystrophy (POLR3A, POLR3B, and POLR1C), respectively. ${ }^{21-24}$ Similarly, clinical and radiologic improvement over time are suggestive of transient infantile HLD19 (TMEM63A). ${ }^{25}$ As illustrated by the expanded phenotyping of these 2 affected individuals with the EIF2AK2-related LEUDEN syndrome, the radiographic findings and neurologic regression with febrile illness are cardinal findings that distinguish it from PMD and other HLDs.

\section{Study Funding}

No targeted funding reported.

\section{Disclosure}

Go to Neurology.org/NG for full disclosures.

\section{Publication History}

Received by Neurology: Genetics July 26, 2020. Accepted in final form October 16, 2020.

\section{Appendix Authors}

\begin{tabular}{|c|c|c|}
\hline Name & Location & Contribution \\
\hline $\begin{array}{l}\text { Daniel G. } \\
\text { Calame, } \\
\text { MD, PhD }\end{array}$ & $\begin{array}{l}\text { Baylor College of } \\
\text { Medicine, Houston, } \\
\text { TX }\end{array}$ & $\begin{array}{l}\text { Major role in acquisition and } \\
\text { analysis of data and drafted the } \\
\text { manuscript }\end{array}$ \\
\hline $\begin{array}{l}\text { Meagan } \\
\text { Hainlen, MD }\end{array}$ & $\begin{array}{l}\text { UT Southwestern } \\
\text { Medical Center, } \\
\text { Houston, TX }\end{array}$ & $\begin{array}{l}\text { Acquisition of data and revised } \\
\text { the manuscript critically }\end{array}$ \\
\hline $\begin{array}{l}\text { Danielle } \\
\text { Takacs, MD }\end{array}$ & $\begin{array}{l}\text { Baylor College of } \\
\text { Medicine, Houston, } \\
\text { TX }\end{array}$ & $\begin{array}{l}\text { Acquisition of data and revised } \\
\text { the manuscript critically }\end{array}$ \\
\hline $\begin{array}{l}\text { Leah } \\
\text { Ferrante, } \\
\text { MD }\end{array}$ & $\begin{array}{l}\text { Baylor College of } \\
\text { Medicine, Houston, } \\
\text { TX }\end{array}$ & $\begin{array}{l}\text { Acquisition of data and revised } \\
\text { the manuscript critically }\end{array}$ \\
\hline $\begin{array}{l}\text { Kayla } \\
\text { Pence, MD }\end{array}$ & $\begin{array}{l}\text { Baylor College of } \\
\text { Medicine, Houston, } \\
\text { TX }\end{array}$ & $\begin{array}{l}\text { Acquisition of data and revised } \\
\text { the manuscript critically }\end{array}$ \\
\hline $\begin{array}{l}\text { Lisa T. } \\
\text { Emrick, MD }\end{array}$ & $\begin{array}{l}\text { Baylor College of } \\
\text { Medicine, Houston, } \\
\text { TX }\end{array}$ & $\begin{array}{l}\text { Acquisition of data and revised } \\
\text { the manuscript critically }\end{array}$ \\
\hline
\end{tabular}

Appendix (continued)

\begin{tabular}{lll}
\hline Name & Location & Contribution \\
\hline Hsiao-Tuan & Baylor College of & Major role in acquisition and \\
Chao, MD, & Medicine, Houston, & $\begin{array}{l}\text { analysis of data and revised the } \\
\text { manuscript critically }\end{array}$ \\
PhD & TX &
\end{tabular}

\section{References}

1. Bonkowsky JL, Nelson C, Kingston JL, Filloux FM, Mundorff MB, Srivastava R. The burden of inherited leukodystrophies in children. Neurology 2010;75: $718-725$.

2. Osório MJ, Goldman SA. Neurogenetics of Pelizaeus-Merzbacher disease. Handbook Clin Neurol 2018;148:701-722.

3. van der Knapp MS, Schiffmann R, Mochel F, Wolf NI. Diagnosis, prognosis, and treatment of leukodystrophies. Lancet Neurol 2019;18:962-972.

4. Inoue K. Pelizaeus-Merzbacher disease: molecular and cellular pathogenesis and associated phenotypes. Adv Exp Med Biol 2019;1190:201-216.

5. Schiffmann R, van der Knaap MS. Invited article: an MRI-based approach to the diagnosis of white matter disorders. Neurology 2009;72:750-759.

6. Numata Y, Gotoh L, Iwaki A, et al. Epidemiological, clinical, and genetic landscapes of hypomyelinating leukodystrophies. J Neurol 2014;261:752-758.

7. Ji H, Li D, Wu Y, et al. Hypomyelinating disorders in China: the clinical and genetic heterogeneity in 119 patients. PLoS One 2018;13:e0188869.

8. Vanderver A, Bernard G, Helman G, et al. Randomized clinical trial of first-line genome sequencing in pediatric white matter disorders. Ann Neurol 2020;88: 264-273.

9. Gupta N, Henry RG, Kang S, et al. Long-term safety, immunologic response, and imaging outcomes following neural stem cell transplantation for PelizaeusMerzbacher disease. Stem Cell Rep 2019;13:254-261.

10. Mao D, Reuter CM, Ruzhnikov MRZ, et al. De novo EIF2AK1 and EIF2AK2 variants are associated with developmental delay, leukoencephalopathy, and neurologic decompensation. Am J Hum Genet 2020;106:570-583.

11. Lek M, Karczewski KJ, Minikel EV; Karczewski, Exome Aggregation Consortium, et al. Analysis of protein-coding genetic variation in 60,706 humans. Nature 2016;536: 285-291.

12. Karczewski KJ, Francioli LC, Tiao G; Genome Aggregation Database (gnomAD) Consortium, et al. The mutational constraint spectrum quantified from variation in 141,456 humans. Nature 2020;581:434-443.

13. Richards S, Aziz N, Bale S, et al. Standards and guidelines for the interpretation of sequence variants: a joint consensus recommendation of the American College of medical genetics and genomics and the association for molecular pathology. Genet Med 2015; 17:405-424

14. Wek R, Jiang H, Anthony TG. Coping with stress: eIF2 kinases and translational control. Biochem Soc Trans 2006;34:7-11.

15. Acharya P, Chen J, Correira M. Hepatic heme-regulated inhibitor (HRI) eukaryotic initiation factor 2alpha kinase: a protagonist of heme-mediated translational control of CYP2B enzymes and a modulator of basal endoplasmic reticulum stress tone. Mol Pharmacol 2010:77575-77592.

16. Ito T, Yang M, May W. RAX, a cellular activator for double-stranded RNA-dependent protein kinase during stress signaling. J Biol Chem 1999;274:15427-15432.

17. Williams B. PKR; a sentinel kinase for cellular stress. Oncogene 1999;18:6112-6120.

18. Galluzzi L, Brenner C, Morselli E, Touat Z, Kroemer G. Viral control of mitochondria apoptosis. PLoS Pathog 2008;4:e1000018.

19. Bugiana M, Boor I, Powers JM, Scheper GC, van der Knaap MS. Leukoencephalopathy with vanishing white matter: a review. J Neuropathol Exp Neurol 2010;69:987-996.

20. Vanderver A, Prust M, Tonduti D, et al. Case definition and classification of leukodystrophies and leukoencephalopathies. Mol Genet Metab 2015;114:494-500.

21. Mierzewska H, Rydzanicz M, Bieganski T, et al. Spondyloepimetaphyseal dysplasia with neurodegeneration associated with AIFM1 mutation-a novel phenotype of the mitochondrial disease. Clin Genet 2016;91:30-37.

22. Zara F, Biancheri R, Bruno C, et al. Deficiency of hyccin, a newly identified membrane protein, causes hypomyelination and congenital cataract. Nat Genet 2006;38: 1111-1113.

23. Gauquelin L, Cayami FK, Sztriha L, et al. Clinical spectrum of POLR3-related leukodystrophy caused by biallelic POLR1C pathogenic variants. Neurol Genet 2019;5:e369.

24. Thiffault I, Wolf NI, Forget D, et al. Recessive mutations in POLR1C cause a leukodystrophy by impairing biogenesis of RNA polymerase III. Nat Commun 2015;6: 7623.

25. Yan H, Helman G, Murthy SE, et al. Heterozygous variants in the mechanosensitive ion channel TMEM63A result in transient hypomyelination during infancy. Am J Hum Genet 2019;105:996-1004. 


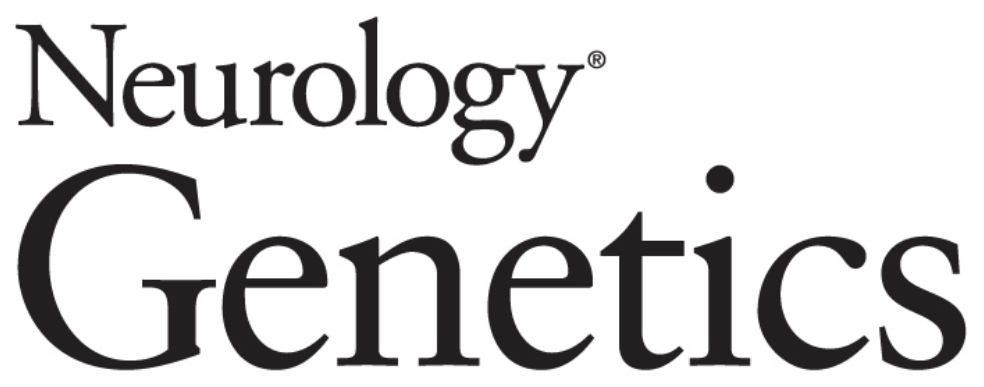

\section{EIF2AK2-related Neurodevelopmental Disorder With Leukoencephalopathy, Developmental Delay, and Episodic Neurologic Regression Mimics Pelizaeus-Merzbacher Disease}

Daniel G. Calame, Meagan Hainlen, Danielle Takacs, et al. Neurol Genet 2021;7;

DOI 10.1212/NXG.0000000000000539

This information is current as of December 17, 2020

Neurol Genet is an official journal of the American Academy of Neurology. Published since April 2015, it is an open-access, online-only, continuous publication journal. Copyright Copyright ( 2020 The Author(s). Published by Wolters Kluwer Health, Inc. on behalf of the American Academy of Neurology.. All rights reserved. Online ISSN: 2376-7839.

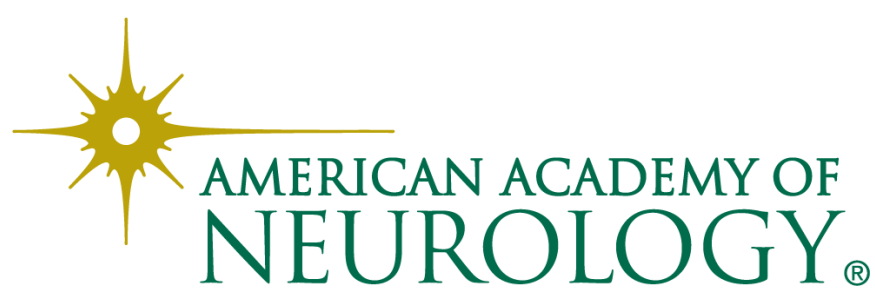




\section{Updated Information \& Services}

References

Subspecialty Collections

Permissions \& Licensing

Reprints including high resolution figures, can be found at: http://ng.neurology.org/content/7/1/e539.full.html

This article cites 24 articles, 3 of which you can access for free at: http://ng.neurology.org/content/7/1/e539.full.html\#\#ref-list-1

This article, along with others on similar topics, appears in the following collection(s):

\section{All Genetics}

http://ng.neurology.org//cgi/collection/all_genetics

Developmental disorders

http://ng.neurology.org//cgi/collection/developmental_disorders

Leukodystrophies

http://ng.neurology.org//cgi/collection/leukodystrophies

Nystagmus

http://ng.neurology.org//cgi/collection/nystagmus

Information about reproducing this article in parts (figures,tables) or in its entirety can be found online at:

http://ng.neurology.org/misc/about.xhtml\#permissions

Information about ordering reprints can be found online: http://ng.neurology.org/misc/addir.xhtml\#reprintsus

Neurol Genet is an official journal of the American Academy of Neurology. Published since April 2015, it is an open-access, online-only, continuous publication journal. Copyright Copyright $\odot 2020$ The Author(s). Published by Wolters Kluwer Health, Inc. on behalf of the American Academy of Neurology.. All rights reserved. Online ISSN: 2376-7839.

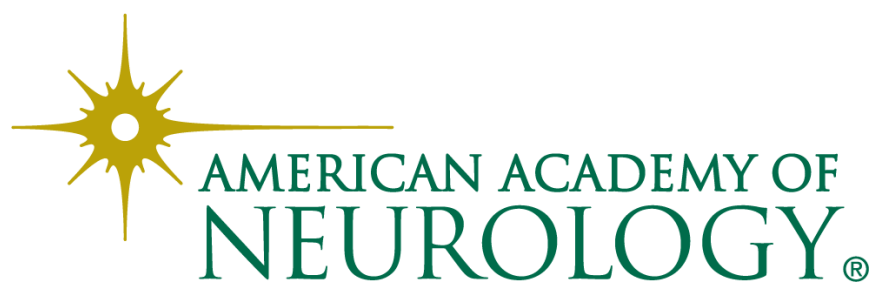

